\title{
A Near Infrared Spectroscopy System for Assessing Rehabilitation on Peripheral Arterial Occlusion Patients
}

\author{
Shao-Hung Lu ${ }^{1} \cdot$ Cheng-Lun Tsai ${ }^{1} \cdot$ Tieh-Cheng Fu ${ }^{2} \cdot$ Po-Hung Chang ${ }^{1} \cdot$ Shao-Ming Lu ${ }^{3}$
}

Received: 23 May 2020 / Accepted: 6 July 2020 / Published online: 28 July 2020

(c) The Author(s) 2020

\begin{abstract}
Purpose A near-infrared spectroscopy system was developed to evaluate peripheral arterial occlusive disease (PAOD). The wireless device can take measurements when patients are doing rehabilitation exercise.

Methods Two LED modules with wavelengths of $740 \mathrm{~nm}, 808 \mathrm{~nm}$ and $850 \mathrm{~nm}$ were placed over $32 \mathrm{~mm}$ away from the photodiode to detect the deep backscattering from muscle tissue. The absorbance difference from the extent of tissue oxygenation was extracted using a modified Beer's law. Measurements taken on different muscle groups were applied to determine which branch of artery is occluded.

Results The changes in oxygenation of gastrocnemius and tibialis anterior muscle were recorded when PAOD patients were riding a stationary bicycle. The drop in oxygenation with exercise generally should recover within 15 min of continuing exercise. Otherwise, the artery is not capable to provide enough blood supply to the exercising muscles. Since gastrocnemius is the primary muscle used in biking exercise, the compensatory vasodilation in gastrocnemius leads to hypoperfusion in tibialis anterior muscle.

Conclusion This NIRS system is helpful for detecting the changes in tissue oxygenation during exercise. Muscle hypoxia was more obvious during exercise when the artery had some degree of occlusion.
\end{abstract}

Keywords Peripheral arterial occlusive disease, PAOD $\cdot$ Near-infrared spectroscopy $\cdot$ Tissue oxygenation $\cdot$ Rehabilitation exercise

\section{Introduction}

Peripheral arterial occlusive disease (PAOD) has a very high prevalence rate among elderly people. Its occurrence in the lower extremities causes leg pain and leads to difficulty walking. A patient who is unable to walk will become a heavy burden on his/her family. An aging society with a lot of PAOD patients not only increases medical costs but also results in financial problems of a country. The best way to reduce patient's pain and medical costs is through early

Cheng-Lun Tsai

clt@cycu.edu.tw

1 Biomedical Engineering Department of Chung Yuan Christian University, 200 Chung-Pei Road, Chung-Li District, Taoyuan City 32023, Taiwan

2 Department of Physical Medicine and Rehabilitation, Chang Gung Memorial Hospital, Keelung, Taiwan

3 Office of Epidemiology and Biostatistics, Koo Foundation-Sun Yat-Sen Cancer Center, Taipei, Taiwan detection to improve the conditions at the reversible stage $[1,2]$.

Ankle-Brachial pressure index (ABI) is the standard clinical detecting method of peripheral arterial occlusion disease at present. The ABI is performed by measuring the peak systolic pressure of the ankle and brachial arteries using air cuffs on each limb. The ABI value is calculated by dividing the ankle arterial systolic pressure by the brachial arterial systolic pressure. Following the 2016 AHA/ACC Guideline, the normal range of this index falls between 0.90 and 1.40. The index value between 0.4 and 0.9 indicates moderate or some arterial diseases. The value less than 0.4 represents severe arterial diseases [3].

The ABI measurement has to be taken in the supine position; in addition, an experienced physician is required for the blood pressure measurement and interpretation. This makes it difficult to promote a community-wide screening of peripheral arterial occlusion in the geriatric population for early treatments. Generally, PAOD patients will not go to a hospital for examinations and treatments until they feel 
too painful to walk. If a simple early detection method for PAOD can be developed, it will reduce the medical costs and improve life quality of the PAOD patients. Near-infrared spectroscopy (NIRS) is an optical measurement method using multiple light wavelengths in the near-infrared range. It is widely used to measure the blood perfusion and the oxygenation of biological tissue non-invasively $[4,5]$. NIRS was also shown to detect the increase of blood transfusion and oxygenation on PAOD and cardiovascular patients during rehabilitation exercise [6, 7].

In this study, an NIRS device developed to measure oxygenation in muscle tissue during rehabilitation exercise was proposed as an accessing method for PAOD. PAOD patients undertaking rehabilitation exercise were recruited as subjects in this study. Blood flow through artery in muscle tissue will greatly increase during exercise to meet the high oxygen demand. Therefore, the abnormal changing pattern of tissue oxygenation during exercise has a great potential to identify the development of arterial occlusion at its early stage. The NIRS system also has the advantages of low cost, being small, light and easy to use. These advantages are of great help to develop an NIRS PAOD screening instrument for clinical practice [8].

\section{Methods}

\subsection{Oxygenation in Muscle Tissue}

Near-infrared absorption spectra of biological tissue are widely used to quantify the changes in tissue compositions of biological tissue. Near-infrared light can travel in biological tissue for a relatively long distance because of their low near-infrared absorption properties. In this study, an NIRS optical probe was designed to detect the tissue oxygenation in deep muscle tissue. To ensure the light would travel through muscle tissue, light source and detector were separated by a distance greater than the detecting depth. Only a small portion of the light that penetrates through skin and fat tissues was scattered from the underneath muscle tissue back to the skin surface and detected by the photo sensor. If two light sources are closely spaced at different distances away from the photo sensor, the detected light from two sources will travel in the same tissue region through nearly the same path. However, the reflectance from the far source travels slightly longer in muscle tissue $(\Delta L)$ than it from the near one. By taking the difference of reflectance between two sources, the light absorbed in skin and fat tissues from two light paths cancel out each other. This is similar to treat the near reflectance as an incident light and the far reflectance as the transmitted light, then the small portion of absorbance $\left(\Delta A_{\Delta L}\right)$ in muscle tissue can be extracted. Equation (1) shows how the difference of absorbance is calculated by taking the logarithm of the ratio of the near reflectance intensity $(R n)$ to the far reflectance intensity $(R f)[9]$.

$\Delta A_{\Delta L}=\log \frac{R_{n}}{R_{f}}$

The absorbance difference in muscle tissue is further assumed to be contributed by myoglobin in muscle tissue and hemoglobin in red blood cells as well as the scattering attenuation in muscle tissue. Myoglobin has a stronger oxygen affinity than that of hemoglobin, but their absorption spectra have similar spectral profile [10]. Since it is difficult to distinguish the contribution of myoglobin and hemoglobin on tissue oxygenation, the oxygenation of two tissue constituents were lumped together as tissue oxygenation.

Equation (2) is the modified Beer's law equation to quantify the contribution of hemoglobin and oxyhemoglobin. The original Beer's law is a basic tool to separate absorbance by different chromophores in a translucent sample solution. Since biological tissue is generally opaque with strong light scattering property, a baseline shift is usually found in tissue absorbance spectra. A scattering term can be added to modify the Beer's law equation for matching the absorbance shift in an opaque sample [11]. The absorbance in Eq. (2) includes the absorption by oxygenated tissue and deoxygenated tissue as well as the scattering by tissue, where $a_{t} O$ is the absorption coefficient of oxygenated muscle tissue, $a_{t}$ is the absorption coefficient of deoxygenated muscle tissue, and $s_{t}$ is the scattering coefficient of tissue. The absorption coefficients of hemoglobin derivatives were used as those of tissue in the calculation. Since blood perfusion rate in muscle tissue greatly increases with exercise, blood volume also have great contribution to the change in light absorbance during the measurement. The average path length in oxygenated part of muscle tissue is represented by $l_{t o}, l_{t}$ represents the average path length in the part of deoxygenated muscle tissue, and $l_{s}$ represents the average path length that is not affected by oxygenation in muscle tissue. The change in oxygenation level of muscle tissue is quantified by tracking the $l_{t O}$ value.

Muscle tissue with normal blood supply can easily maintain the oxygenation of myoglobin at a high level during moderate exercise. In the muscle tissue of PAOD patients where artery is moderately or severely occluded, muscle tissue cannot receive enough oxygen supply through blood perfusion. The limited blood flow results in a great drop of tissue oxygenation during exercise that finally makes patient feel muscle pain.

$\Delta A_{\Delta L}=a_{t o} l_{t O}+a_{t} l_{t}+s_{t} l_{s}$

There are three unknown variables in this linear equation, therefore the absorbance have to be measured at no less than three wavelengths in order to solve these three variables. 


\subsection{NIRS Optical Probes and Measurement System}

The light source in this near-infrared spectroscopic system was LEDs with three close wavelengths: $740 \mathrm{~nm}, 808 \mathrm{~nm}$, $850 \mathrm{~nm}$. The scattering path of light traveling in biological tissue is determined by the scattering coefficient of the tissue. By choosing LEDs with similar wavelengths, their paths in tissue would be relatively the same. To be more sure that these wavelengths would propagate through the same path in tissue, LEDs were packed together in a small customized 5050 package module. The distance among LED dices were only 1 to $2 \mathrm{~mm}$. These wavelengths were also chosen because the central wavelength at $808 \mathrm{~nm}$ was almost identical with the isosbestic point of hemoglobin and oxyhemoglobin absorption spectra around $805 \mathrm{~nm}$. The transmission intensity at this wavelength will not change with the oxygen saturation of hemoglobin and myoglobin. Only a change in tissue blood volume would cause a change in light transmittance of $808 \mathrm{~nm}$. The difference of absorption coefficient among three wavelengths was also relatively small. This kept their transmission intensity in a similar range which was helpful in the design of detecting circuit. Two LED modules spaced $8 \mathrm{~mm}$ from center to center were used as the near and the far light sources. Six LEDs emit light in a time-divided sequence were controlled by a System-on-aChip (SoC) microprocessor (HY16F198, Hycon Tech. Inc.). The backscattering reflectance is picked up by a photodiode (BPW34, VISHAY) sitting $32 \mathrm{~mm}$ away from the center of the near LED module, see Fig. 1. The driving current of each near and far LEDs were adjusted by placing inside a large integrating sphere to have the same emission intensity.

The backscattered light from tissue was converted to voltage signal by a trans-impedance amplifier (TIA); then was digitized by a built-in 24 bits sigma-to-delta analogto-digital converter in the SoC microprocessor. The digital signal of light intensity of each wavelength for the near and far LED modules was synchronously resolved with their driving current pulses. The signals acquired by each optical probe were all gathered by the main circuit module. The main circuit module can connect up to four NIRS optical probes. Finally, the continuously measured reflectance signals were wirelessly transmitted to a laptop computer through Bluetooth communication from the main circuit module. Further analysis of spectra were carried out and recorded in the computer. During the experiment, physicians can monitor the signal traces displayed on computer screen, as the block diagram shown in Fig. 2.

Anterior tibial artery and posterior tibial artery are arteries that supply blood flow to two major muscle groups in lower legs: tibialis anterior muscle and gastrocnemius muscle, respectively. ABI measurement can only tell that the arterial occlusion is on the right leg or the left leg, but no information could show which artery is occluded. A further examination using Doppler ultrasound or Magnetic Resonance Imaging is generally required to find the real occluded artery.

Four NIRS optical probes were used in this study by attaching to two major muscle groups of both lower legs. The RA probe was placed on the tibialis anterior muscle, and RP probe was placed on the medial gastrocnemius muscle of the right lower leg. The LA and LP probes were placed on the left lower leg. By measuring the changes in tissue oxygenation during the rehabilitation exercise, these probes were used to find which muscle had the sign of artery occlusion.
Fig. 1 Cross section view of tissue showing the mean paths of scattering reflectance from two separate LED light source to the photodiode

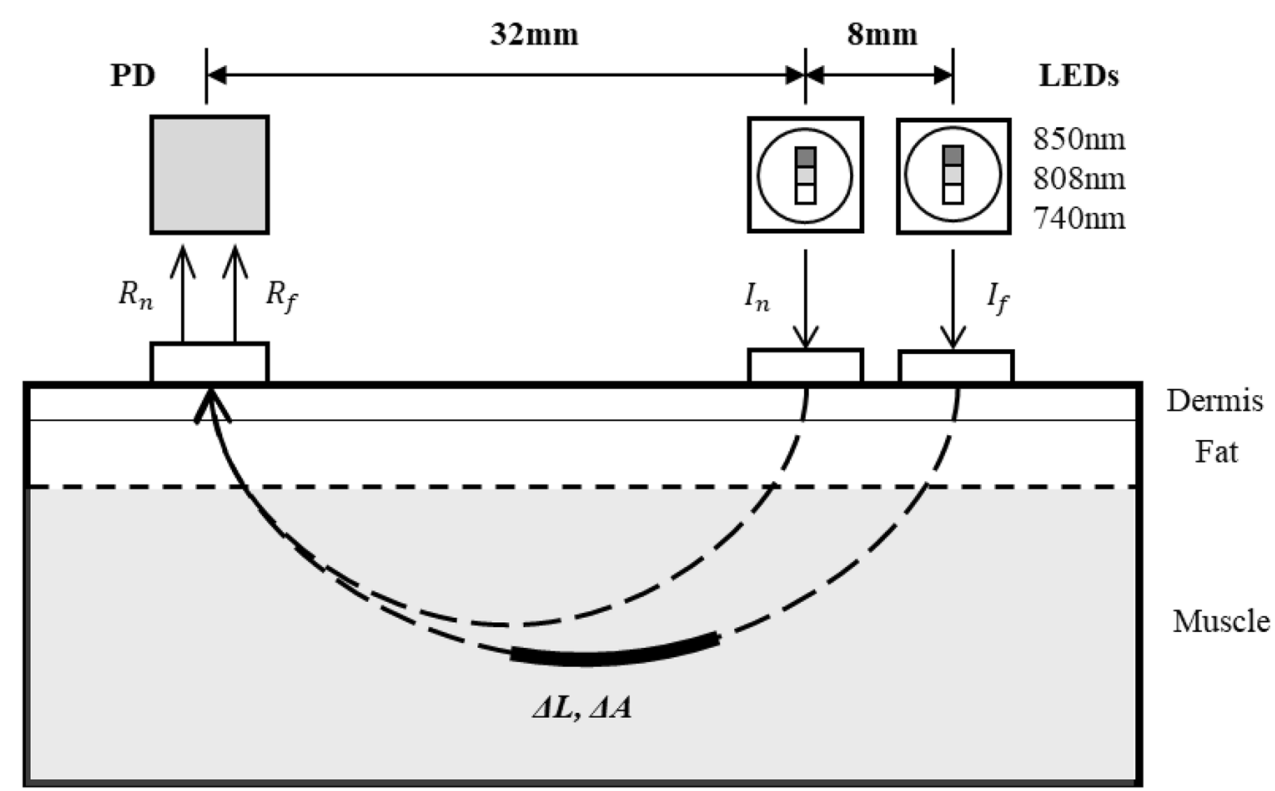




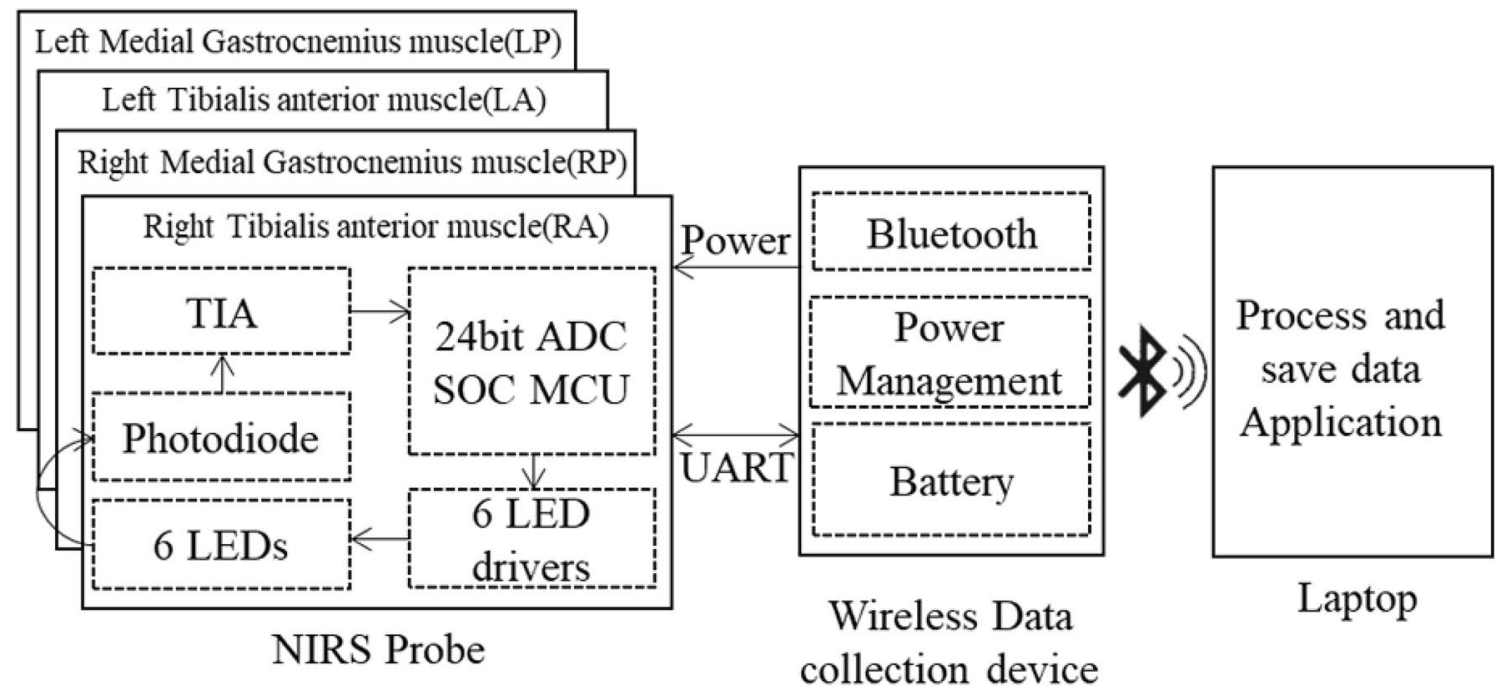

Fig. 2 Block diagram of the NIRS measurement system

\subsection{Rehabilitation Evaluation}

Thirteen outpatients who had been diagnosed with peripheral arterial occlusion using ankle-brachial index (ABI) at the Heart Failure Center of Chang Gung Memorial Hospital were recruited in this study. These patients experienced leg pain during walking, and undertook ABI measurement. MRI exam was also used to confirm the presence of occlusion before the operation. Once the occlusion was confirmed, peripheral angioplasty was suggested if the patient cannot endure the leg pain. All the patients were undertaking a rehabilitation exercise with a stationary bicycle during this study. This near-infrared spectroscopy device was also used as an assessment of the therapeutic exercise.

Before the therapeutic exercise training, patients performed a supervised cardiopulmonary exercise test on a bicycle ergometer (Ergoselect 150P, ergoline $\mathrm{GmbH}$ ) to find their peak exercise workload and oxygen consumption. During this test, the workload was stepped up $10 \mathrm{~W}$ per minute until the patients felt exhausted. The rehabilitation exercise intensity of ergometer was set at half of this peak exercise power. Four NIRS optical probes were carefully placed and fixed on patient's lower legs to record the changes in tissue oxygenation while riding a bicycle ergometer.

Right before the initiation of exercise, signals were recorded at rest for $5 \mathrm{~min}$ as the reference tissue oxygenation level. Then, the patient started to ride the bicycle ergometer for up to $30 \mathrm{~min}$. If the patient felt too painful to continue, they could slow down or stop whenever they want to. The real time signal traces were monitored by the assistant while carrying on the rehabilitation exercise and saved in a computer for further analysis. The measurement is to evaluate the dynamic changes of oxygen supply to the working muscles to assess the condition of their supplying arteries. This study was approved by the Institutional Review Board of Chang Gung Memorial Hospital in Taiwan.

\section{Results}

Figure 3 shows the bottom view of a NIRS optical probe built for this study, and how a patient wears them during the measurement. The long distances between the LED modules and photodetector ensure the light to have travelled deep enough, about 10 to $20 \mathrm{~mm}$, into the muscle tissue before arriving the photodiode.

The clinical characteristics of patients involved in this study are shown in Table 1. Outpatients with leg pain were diagnosed by doctors using ABI air cuff blood pressure measurement. The ABI index values of two legs were list separately. Patients' artery occlusion confirmed by the MRI exam was marked with closed circles, and with open circles for the patents. Black triangles indicate the arteries undertook balloon angioplasty before rehabilitation exercise. Patients who had balloon angioplasty at least in one leg were grouped together and listed at the top. These patients had the most serious occlusion found in the arteries. Patients with moderate occlusion and did not have balloon angioplasty were list at the end.

The sample curves of $\boldsymbol{l}_{\boldsymbol{t}}$ through the rehabilitation exercise of two patients are shown in Figs. 4 and 5. Once the biking exercise started, the $\boldsymbol{l}_{\boldsymbol{t}}$ value generally has a sudden drop within a minute. This value would drift slowly back to the starting value in $15 \mathrm{~min}$, if compensation dilation can provide enough blood supply, as shown in Fig. 4. In the NIRS 


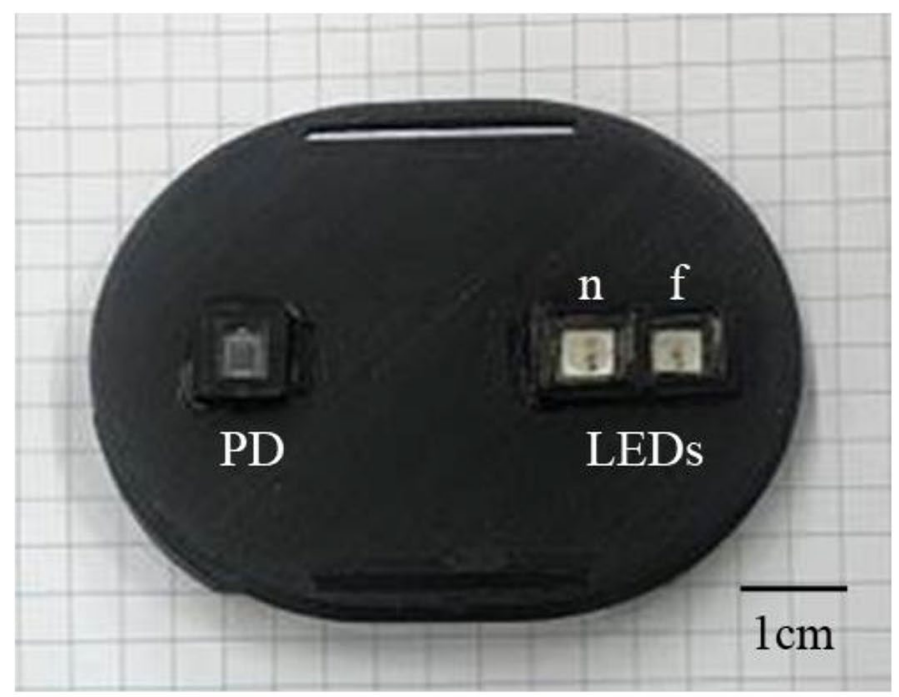

(a)

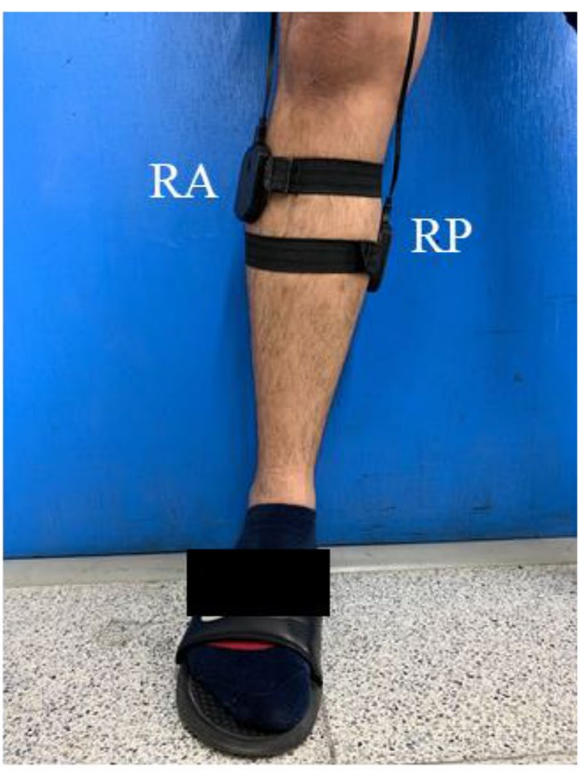

(b)

Fig. 3 a Bottom view of NIRS optical probe and $\mathbf{b}$ the measurement sites on right lower leg

Table 1 Patient clinical characteristics

\begin{tabular}{|c|c|c|c|c|c|c|c|c|c|c|c|c|c|}
\hline \multirow{2}{*}{ Subject } & \multirow{2}{*}{ Gender } & \multirow{2}{*}{ Age } & \multirow{2}{*}{$\begin{array}{l}\text { Peak exercise Load(W)/ } \\
\text { Rehabilitation Load(W) }\end{array}$} & \multirow{2}{*}{$\begin{array}{l}\text { ABI } \\
\text { (R) }\end{array}$} & \multirow{2}{*}{$\begin{array}{l}\text { ABI } \\
\text { (L) }\end{array}$} & \multicolumn{4}{|c|}{ Diagnosis } & \multicolumn{4}{|c|}{$\operatorname{NIRS}\left(\Delta l_{t o}\right)$} \\
\hline & & & & & & RA & $\mathbf{R P}$ & LA & LP & RA & $\mathbf{R P}$ & LA & LP \\
\hline 1 & M & 74 & $90 / 50$ & 0.83 & 0.52 & 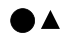 & 01 & 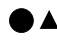 & 1 & -21.8 & -18.0 & -6.7 & -5.6 \\
\hline 2 & M & 67 & $110 / 60$ & 1 & 1.05 & $O \Delta$ & 0 & $\mathbf{O}$ & 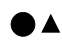 & -13.7 & -6.1 & -16.3 & -6.6 \\
\hline 3 & M & 64 & $80 / 40$ & 0.61 & 0.76 & 0 & $0 \Delta$ & 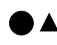 & 01 & -13.2 & 1.0 & -2.2 & -0.2 \\
\hline 4 & M & 70 & $90 / 50$ & 0.87 & 0.53 & $O \Delta$ & $0 \Delta$ & 01 & 01 & -14.1 & 2.1 & -12.4 & 0.8 \\
\hline 5 & $\mathrm{~F}$ & 70 & $50 / 30$ & 0.84 & 0.77 & $O \Delta$ & $O \Delta$ & $\mathbf{O A}$ & 1 & -9.5 & -0.1 & -4.9 & -1.6 \\
\hline 6 & M & 68 & $50 / 30$ & 0.72 & 0.75 & $O \Delta$ & $O \Delta$ & 01 & 01 & -0.9 & 0.0 & -22.4 & 12.4 \\
\hline 7 & $\mathrm{~F}$ & 77 & $60 / 30$ & 0.97 & 1.06 & $O \Delta$ & $0 \Delta$ & 0 & 01 & -9.1 & 0.8 & -8.3 & -6.4 \\
\hline 8 & M & 55 & $80 / 40$ & 0.77 & 1.07 & 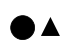 & 01 & 0 & 0 & -0.4 & 4.5 & -16.8 & 3.4 \\
\hline 9 & M & 65 & $120 / 60$ & 1.19 & 1.05 & 0 & 0 & 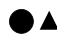 & 04 & -3.0 & -0.4 & -14.7 & 4.8 \\
\hline 10 & M & 70 & $80 / 40$ & 0.88 & 0.89 & 0 & 0 & 0 & 0 & -18.9 & -0.3 & -16.1 & 1.4 \\
\hline 11 & M & 78 & $50 / 30$ & 0.63 & 1.1 & 0 & 0 & 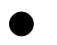 & 0 & -16.9 & -2.1 & -20.2 & 2.9 \\
\hline 12 & M & 79 & $60 / 30$ & 1.06 & 0.92 & 0 & 0 & 0 & 0 & 12.2 & 6.9 & 17.7 & -1.4 \\
\hline 13 & M & 60 & $110 / 50$ & 0.88 & 0.77 & 0 & 0 & 0 & 0 & -7.7 & -4.8 & -5.3 & -11.4 \\
\hline
\end{tabular}

column, the condition of arteries are judged by the recovery value $\left(\Delta l_{t o}\right)$, which is the difference between the value after $1 \mathrm{~min}$ of exercise $\left(\boldsymbol{l}_{\boldsymbol{t} O 1}\right)$ and after $15 \mathrm{~min}$ of exercise $\left(\boldsymbol{l}_{\boldsymbol{t} O \mathbf{1 5}}\right)$, as calculated using Eq. (3).
$\Delta l_{t o}=l_{t o 15}-l_{t o 1}$

Measurements with a $\Delta l_{t o}$ value greater than zero meant the artery in muscle was capable to supply enough oxygen 


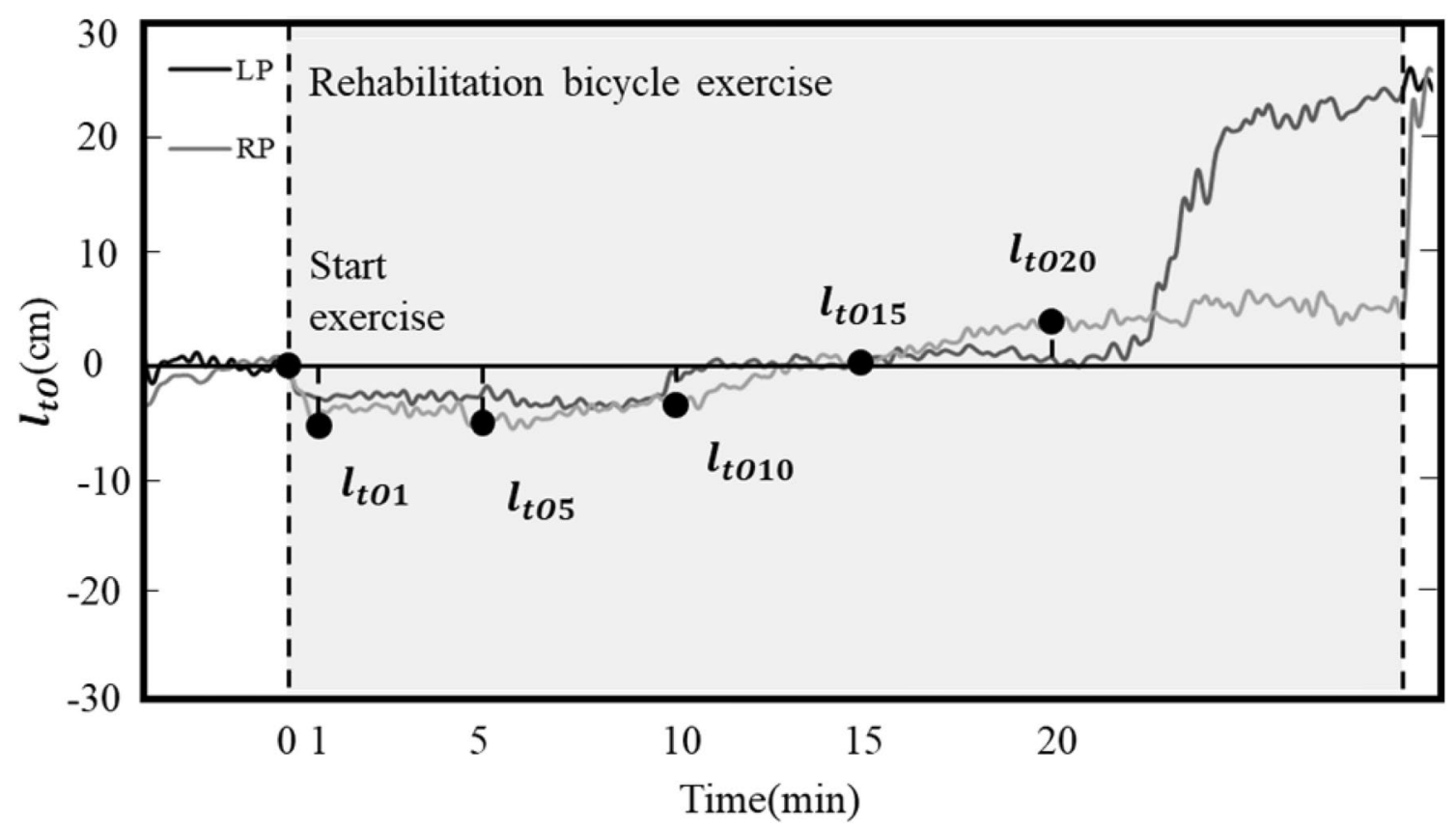

Fig. 4 The $l_{t o}$ curves of LP and RP of subject 8 during biking exercise. The closed circles shows the $l_{t o}$ of RA at 1, 5, 10, 15 and 20 min after starting exercise

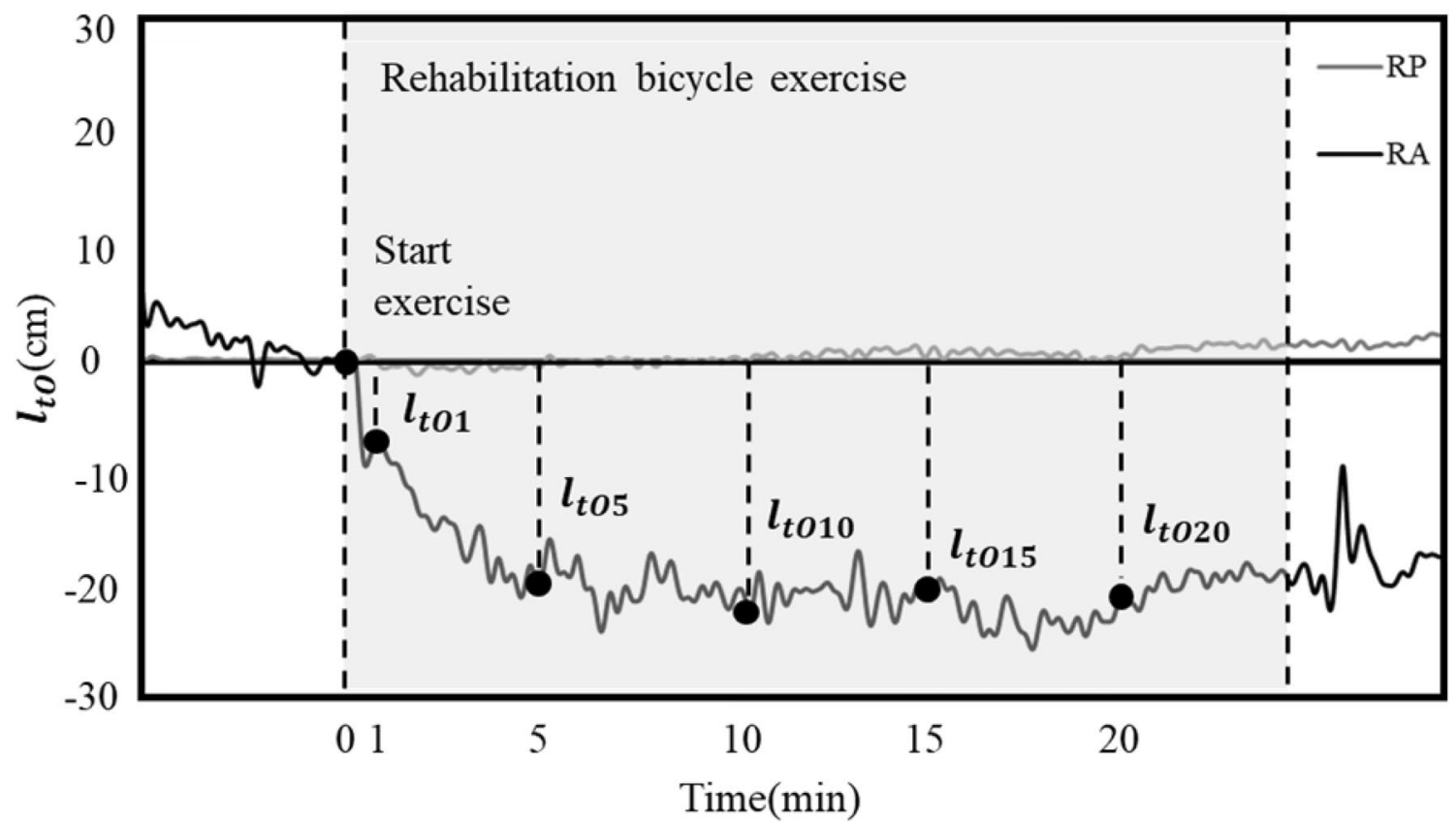

Fig. 5 The $l_{t o}$ curves of RP and RA of subject 7 during biking exercise. The closed circles shows the $l_{t o}$ of RA at 1, 5, 10, 15 and 20 min after starting exercise

for the rehabilitation exercise. The measurements were classified as having a patent artery. The classification is marked with white background in the NIRS column of Table 1. Measurements with a negative $\Delta l_{t} \boldsymbol{v}$ value were classified as having an occluded artery. The negative values in the table were emphasized with grey background.

Every patient received four measurements on two lower legs: RA, RP, LA and LP. The results of all 52 measurements are plotted as a scattering graph in Fig. 6. The horizontal 
axis is the $\boldsymbol{l}_{t o 1}$ value, and the vertical axis is the $\Delta \boldsymbol{l}_{t \boldsymbol{O}}$ value. Generally, a patent artery is able to recover from the $\boldsymbol{l}_{t o 1}$ drop within $15 \mathrm{~min}$ and have a positive $\Delta l_{t o}$ value. The $\Delta l_{t o}$ with positive values are marked with open circles, and the negative ones are marked with closed circles.

\section{Discussion}

All patients went to see a doctor because of leg pain during walking, and were diagnosed with different severity of peripheral arterial occlusion. The doctor's diagnosis is based on not only the ABI values but also patients' own feeling of pain during walking, and finally confirmed by the MRI exam. Four patients $(2,7,9$, and 12) did have ABI values higher than 0.9 on both legs, but they were diagnosed as occluded arteries from the MRI exam. Patients 9 was found to have occlusion in the left lower leg. The right lower leg of patient 10 had ABI value of 0.88 , which is close to the margin. His MRI exam showed no obvious occlusion, and it was classified as patent.

Since the value of ABI does not always correctly reflect the results of MRI exam, the ABI value was compared to doctor's final diagnosis using classification analysis to evaluate the accuracy of ABI. Based on our samples with the 0.9 criteria of 2016 AHA/ACC Guideline, ABI correctly diagnosed PAOD in 15 of 24 doctor diagnosed cases $(62.5 \%$ sensitivity). ABI also identified 1 of 2 legs that were patent (50\% specificity). There was one false-positive result (positive predictive value or precision 93.8\%) and 9 false-negative results (negative predictive value $10 \%$ ). On the whole, ABI

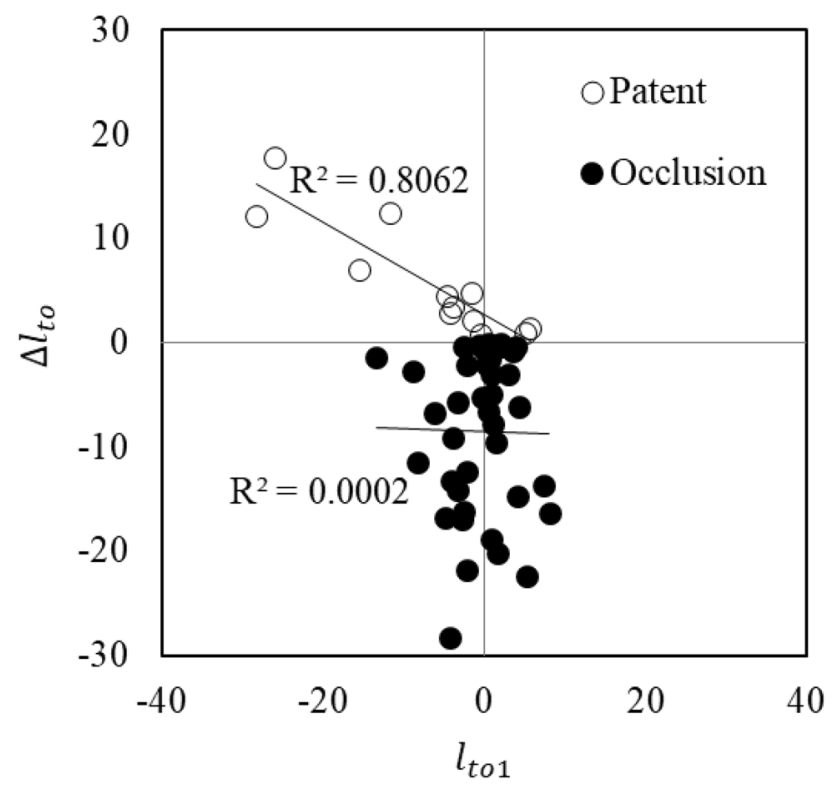

Fig. 6 The $l_{t O 1}$ versus $\Delta l_{t o}$ plot of all 52 measurements diagnosed 16 of all 26 legs correctly (61.5\% accuracy). The values show that $\mathrm{ABI}$ alone is not very accurate for diagnosing PAOD. A second measurement method, such as MRI or Ultrasound imaging, is needed for confirmation.

The ABI method not only has the limitation of taking a static measurement at supine position but also cannot tell which major artery is occluded. MRI exam is more specific in identifying the occluded artery and the location of occlusion. The ABI values of the left legs of patient 7 and 8 showed no peripheral artery occlusion. But, the MRI exam found one of the two major arteries in the lower leg is occluded. This probably means patient's vessel condition is still at an earlier stage, and occlusion just started from one of the arteries in the leg. Therefore, the occlusion is not obvious enough to reflect on the ABI value.

The NIRS measurement was carried out when the patients were doing therapeutic exercise. Most of the recruited patients had already taken the balloon angioplasty. Therefore, the NIRS values are not suitable to compare with the original diagnosis results. The NIRS measurement is mainly used to quantify the dynamic change in tissue oxygenation during the rehabilitation procedure. For normal people at rest, only $15 \%$ to $20 \%$ of cardiac output provides the perfusion in muscle tissue. Under heavy exercise, blood supply to muscle tissue can even rise up over 20 times. If the artery that supplies blood to the muscle is moderate or severely occluded, the oxygen supply to an exercising muscle tissue will not be enough. The oxygen combined with hemoglobin and myoglobin will be very quickly depleted. This is why the oxygenation index $\boldsymbol{l}_{\boldsymbol{t}}$ drops very fast once the exercise is started. To compensate for the loss of oxygen in muscle tissue, blood vessel will dilate to allow more blood to flow into the exercising muscle. The compensatory process will take longer if blood flow in the artery is limited, and hypoxia in muscle tissue will become more obvious. Once the increasing of blood perfusion starts to balance the oxygen consumption of muscle, its oxygenation level gradually rises back to the original level before exercise. It generally takes about 5 to $10 \mathrm{~min}$ for normal people to have oxygenation level totally recovered during moderate exercise. For people with moderate peripheral artery occlusion, it will take longer for the oxygenations level of muscle to recover. The recovery becomes nearly impossible for patient with severe arterial occlusion before stopping exercise. The oxygen level would just stay below the initial level, and the recovery will take a long while even after ceasing the exercise.

The drop of oxygenation level at the first minute after starting exercise was set as the initial value. An artery with proper blood supply capability should be able to compensate the muscle hypoxia at least back to this level during the exercise. A criterion of 15 min was set in this study to classify arterial occlusion. If the oxygen supply cannot reach the equivalent state in $15 \mathrm{~min}$, the condition of artery 
is treated as not able to provide sufficient blood. The NIRS measured not only the changes in muscle tissue oxygenation, but also that in perfusion to muscle tissue. If only the oxygenation was changed, the values of $\boldsymbol{l}_{t} \boldsymbol{a}$ and $\boldsymbol{l}_{\boldsymbol{t}}$ would change in opposite direction while $\boldsymbol{l}_{\boldsymbol{s}}$ remained nearly constant. The real measurements showed the values of $\boldsymbol{l}_{\boldsymbol{t}}$ and $\boldsymbol{l}_{\boldsymbol{t}}$ sometimes both increased or decreased, as well as an obvious change in $\boldsymbol{l}_{\boldsymbol{s}}$ value. These are the evidences of change in tissue blood volume with the constriction or dilation of artery. The changing magnitude of these values are determined by the severity of arterial occlusion [12].

In general, $\Delta l_{t o}$ measured at gastrocnemius muscle is positive or closer to zero than that measured at tibialis anterior muscle. This is because gastrocnemius and soleus in the calf are the major muscles in lower leg used in biking exercise. The anterior tibial artery and posterior tibial artery are both bifurcated from the popliteal artery. The more blood is drained by the posterior tibial artery, the less blood would flow through the anterior tibial artery. Therefore, the negative $\Delta l_{t}$ value is a sign of less blood flow, but it is not necessary mean the anterior tibial artery is really occluded. It merely indicates lower blood perfusion to the tibialis anterior muscle than that is needed during biking exercise. A much longer recovery time would be expected for the tibialis anterior muscle to return its initial state than the gastrocnemius muscle. Other types of exercise would be studied in the future for detecting occlusion in the anterior tibial artery.

The exercise therapy is designed to improve the condition of blood supply by triggering collateral growth of vessel circulation to muscle tissue. Most $\Delta l_{t o}$ values measured at gastrocnemius muscle were positive or close to zero, which means their posterior tibial arteries were relatively wellfunctioning during the exercise. The balloon angioplasty procedure on these occluded arteries seem to achieve the object of treatment. More following studies will try to show whether the exercise therapy is effective to improve performance of the arteries.

\section{Conclusions}

A portable wireless near-infrared spectroscopy device was developed to assess the occlusion of artery in lower legs to help to diagnose the peripheral arterial occlusion disease. Customized LED modules and a System-on-a-Chip microprocessor allow the optical probe to be minimized. The measurement taken during exercise showed a depletion of oxygen and blood in muscle tissue shortly after the beginning of exercise. The tissue oxygenation should be able to recover within $15 \mathrm{~min}$ through increasing the blood supply to muscle tissue. The $\boldsymbol{l}_{\boldsymbol{t}} \boldsymbol{\text { value would stay at lower }}$ level if the arteries could not provide enough blood to the muscle tissue. Such capability to recover tissue oxygenation with 15 min can be used as the sign for identifying the occlusion of artery.

Acknowledgements This research was supported by the Ministry of Science and Technology of Taiwan (MOST 106-2221-E-033-014-, MOST 107-2221-E-033-008), and Chang Gung Medical Research Program (Grant number CMRPG2F0193).

\section{Compliance with Ethical Standards}

Conflict of Interest All contributing authors declare no conflict of interest.

Open Access This article is licensed under a Creative Commons Attribution 4.0 International License, which permits use, sharing, adaptation, distribution and reproduction in any medium or format, as long as you give appropriate credit to the original author(s) and the source, provide a link to the Creative Commons licence, and indicate if changes were made. The images or other third party material in this article are included in the article's Creative Commons licence, unless indicated otherwise in a credit line to the material. If material is not included in the article's Creative Commons licence and your intended use is not permitted by statutory regulation or exceeds the permitted use, you will need to obtain permission directly from the copyright holder. To view a copy of this licence, visit http://creativecommons.org/licenses/by/4.0/.

\section{References}

1. Bess, F., Konrad, J., Paul, N., \& Yvonne, A. (2002). Prevalence of peripheral arterial disease: Persistence of excess risk in former smokers. Australian and New Zealand Journal of Public Health, 26(3), 219-224.

2. Criqui, M. H., \& Aboyans, V. (2015). Epidemiology of peripheral artery disease. Circulation Research, 116(9), 1509-1526.

3. Gerhard-Herman, M. D., Gornik, H. L., Barrett, C., Barshes, R. N., Corriere, M. A., Drachman, D. E., et al. (2016). AHA/ACC guideline on the management of patients with lower extremity peripheral artery disease: A report of the American College of Cardiology/American Heart Association Task Force on Clinical Practice Guidelines. Journal of the American College of Cardiology, 135(12), 726-779.

4. Manfredini, F., Malagoni, A. M., Felisatti, M., Mandini, S., Mascoli, F., Manfredini, R., et al. (2009). A dynamic objective evaluation of peripheral arterial disease by near-infrared spectroscopy. European Journal of Vascular and Endovascular Surgery, 38(4), 441-448.

5. Lamberti, N., Manfredini, F., Tessari, M., Menegatti, E., Nardi, F., Basaglia, N., et al. (2019). A near-infrared spectroscopyassisted test discriminates patients with peripheral arterial disease and venous insufficiency with changes of foot oxygenation following light elastic compression therapy. The European Journal of Vascular Medicine, 48(4), 361-367.

6. Manfredini, F., Malagoni, A. M., Mandini, S., Felisatti, M., Mascoli, F., Basaglia, N., et al. (2012). Near-infrared spectroscopy assessment following exercise training in patients with intermittent claudication and in untrained healthy participants. European Journal of Vascular Endovascular Surgery, 46(4), 315-324.

7. Fu, T. C., Wang, C. H., Lin, P. S., Hsu, C. C., Cherng, W. J., Huang, S. C., et al. (2013). Aerobic interval training improves 
oxygen uptake efficiency by enhancing cerebral and muscular hemodynamics in patients with heart failure. International Journal of Cardiology, 167(1), 41-50.

8. Pellicer, A., \& del Bravo, M. D. C. (2010). Near-infrared spectroscopy: A methodology-focused review. Seminars in Fetal \& Neonatal Medicine, 16(1), 42-49.

9. Lima, A., \& Bakker, J. (2011). Near-infrared spectroscopy for monitoring peripheral tissue perfusion in critically ill patients. Revista Brasileira de Terapia Intensiva, 23(3), 341-351.

10. Park, S. Y., Singh-Moon, R. P., Wan, E. Y., \& Hendon, C. P. (2019). Towards real-time multispectral endoscopic imaging for cardiac lesion quality assessment. Biomedical Optics Express, 10(6), 2829-2846.

11. Maikala, R. V. (2009). Modified Beer's Law-Historical perspectives and relevance in near-infrared monitoring of optical properties of human tissue. International Journal of Industrial Ergonomics, 40(2), 125-134.

12. Kennedy, M. D., Haykowsky, M. J., Boliek, C. A., Esch, B. T., Scott, J. M., \& Warburton, D. E. (2006). Regional muscle oxygenation differences in vastus lateralis during different modes of incremental exercise. Dynamic Medicine, 5, 8. https://doi. org/10.1186/1476-5918-5-8. 